thin iron plate in front of this magnet. These experiments are worth repeating by those who desire clearer conceptions of the manner of action of that remarkable instrument.

\section{SUN-SPOTS AND TERRESTRIAL MAGNETISH}

I $\mathrm{N}$ a remarkable article on "La Météorologie Cosmique," which has appeared in the Annuairc of the Bureau des Longitudes, for $1878, M$. Faye says with reference to the influence of sunspots on the earth's magnetism, that the observations of Cassini "give $17 \delta 7$ " 25 for the date of the maximum observed then at Paris, whilst the latest observations--those of $\mathrm{Mr}$. Broun, himself at Trevandium-assign $3 \delta 70.85$ for the epoch of the last maximum. The interval is 83.60 years. On dividing this by $\delta$, the number of periods in this interval, 10.45 years, are found for the duration of the period. That is to say, almost exactly the value already found by I,amont by means of his own observations at Munich. The period of the spots deduced by M. Wolf, II'I years, not being equal to that for the magnetic variations, these two phenomena have no relation to each other."

I desire to offer a remark on this conclusion, which seems to me too hasty. On examining the two periodic series, that for the diurnal variation of declination and that for the frequency of the solar spots, we sec that there is a perfect coincidence in their phases though the length of successive periods is not constant. We may find a mean length of ten, elesen, or more years, according to the epoch from which the calculation is begun, but we shall always find the same length from both scries if we commence at the same date.

It seems to me then that the true way to determine wheth:r there is an intimate connection between the two Whenomena is to compale their phases, and sce whether the maxima and minima of the one coincide with those of the other. If there is identity in these respects, we must without doubt find the same mean values for the periods.

M. Faye accepts the date $I 787^{\circ 25}$ as that of a maximum for the oscillations of the declination. If we look then at the curve, Fir. 2, given by him in the article in question, we see that this corresponds exactly with a maxirnum of sun-spot frequency. In like manner similar coincidences are seen in the epochs deduced from the obeervations of Arago and others up to the present time when comparcd one by one with the sun-spot observations of Schwabe, Carrington, Secchi, as well as of those mide at Kew. The conclusion seems to me very different from that of M. Faye. We are entitled to apply the rule he bas given (p. 634): "If two series of phenomena, however different they may appear at first, follow exactly the same period, they ought to be referred to the same cause."

There is another passage upon which I desire to offer a remark: "Two kinds of meteors exercise a considerable influence on the direction of the magnetic needle, these are the aurorx boreales and the cyclones."

For the first there is a general agreement, but for the cyclones what observations have we which prove any such influence? If cyclones exercise a considerable influence on the direction of the needle, in what phase of the phenomenon does this occur? Is it on their formation, on their passage over some particular meridian, or when their centre is over a place? In the last case each cyclone will be a source of disturbance, which will be manifested as it progresses, and not simultaneously at all places, which, however, is what really occurs in the case of magnetic disturbances. Electricity is, without doubt, a cause, but only in the case of such considerable discharges as the aurora polaris; but not the local electricity which may accompany cyclones. When there is a storm, and the thunder rolls, and the electrometer shows enormous variations of atmospheric clectricity, changing sign continually, the magnetic needle continues its usual and regular progress. Of this we can offer hundreds of examples.

\section{Lisbon, February 22}

P.S. - The mean movements of the magnetic needle in the Lisbon Observatory, from eight A.M. to two P.M. are given in NATURE, vol. xiii. p. 448 , for the years 1858 to I 875 ; the following are the corresponding mean ranges for the next two years :-
1876
$5.81^{\prime}$
I 877
$54^{\prime}$

So that the mean movement was less in 1877 than in I 876 .

\section{OUR ASTRONOMICAL COLUMN}

New COMPANION TO AldmBaran.-Mr. S. W. Burnham notifies his discovery with the $\mathrm{S} \delta \frac{1}{2}$-inch Alvan Clark refractor of the Dearborn Observatory, of a minute star much nearer to Aldebaran than that which makes the double star H. VI. 66; be compares it with the ruddy bright star, as resembling, in difficulty and appearance, Mars and his outer satellite. The mean of three days' observations gives the angle $109^{\circ} \circ$, and the distance $30^{\prime \prime} \cdot 35$ for $1877^{\circ} 90$, or if the second result which, as printed, differs nearly ten degrees from the other two, the angle will be II I $^{\circ} 9$. The secular proper motion of Aldebaran, according to Mädler, is $19^{\prime \prime} \mathrm{I}$ in the direction $157^{\circ}$; some years must elapse before the question of physical or optical duplicity can be decicled. Eight days' measures of the close companion of Sirius, by Mr. Iurnham, with the same instrument, assign for the angle of position $52^{\circ} \cdot 4$, distance $10^{\prime \prime} \cdot \delta_{j}$ at the epoch 1877.97.

THE STAR LALANDE 378J3.-Mr. J. E. Gore writes from Ballisodare, Co. Sligo, with reference to this star, which appears in the reduced catalogue as a sccond magnitude, and which, observing in the I'unjab in August, I 877 , he had found a little less than Lacaille 8308 or $7 \mathrm{~m}$. This is one of the errors in the catalogue which, as in a case recently noticed in this column, can only be cleared up by referring to the Histoirc Céleste. The observation was made on August 20, 5795 , and the star No. 378r 3 was really estimated $7.8 \mathrm{~m}$. " $\Lambda$ quilæ was observed immediately before it, and entered $2 \mathrm{~m}$.; it is this erroneous magnitude for the bright star of Aquila that has become attached to the star of which Mr. Gore writes. There is a very noticeable proper motion in N.P.D., apparently about $+0^{\prime \prime} 48$ annually, as shown by comparison of the observations of Lacaille, Lalande, Jacob, and Argelander, with the position in the Washington Catalogue for I 860.

The Minor Planets.-Discoveries in this group still progress. No. I 86 was detected by M. Prosper Henry at Paris, on April 6, shining as a star of $11.5 \mathrm{~m}$., and No. I 87 by M. Coggia at Marseilles, on April ro; it was estimated 10 m. No. I78 (Palisa, 1877, November 6) has been named Belisana, and No. I84 (Palisa, I878, February 28) it is proposed to call Deiopeic. With already seven additions to the list, it would not appear that I878 is likely to fall short of the most prolific of preceding years in these discoveries.

The Transit of MERCURy on MAy 6.-If we calculate strictly from Le Verrier's tables of sun and planet, using therefore the value of the sun's diameter which he deduced from the transits of Mercury in his memoir, printed as an addition to the Connaissance des Temps for i 848 , we shall have the following formula for determining the time of the first exterual contact of limbs in the approaching transit:-

$t={ }_{3}$ h. 13 m. Is. $-\left[r^{\circ} 8723\right] r \sin l-\left[I^{\circ} 9079\right] r \cos l, \cos \left(L_{-}^{\circ}-5^{\circ} 49^{\prime} 3\right)$ in which $t$ is the Greenwich mean time of contact, $r$ the 